\title{
Iot based Healthcare System for Coma Patient
}

\author{
Ankita Ramtirthkar, JyothiDigge, V.R.Koli
}

\begin{abstract}
Coma is a state of unconsciousness where the patient fails to respond. These patients need utmost care and 24 *7 observations. This paper presents a continuous monitoring and recording of patient data without human intervention. If there is any sudden changes occur in the normal range of body parameters such as body temp falls or rise, blood pressure (B. P.) increases or decreases causing high or low B.P. where both are not stable conditions for better health, then it has facility to automatically alert the medical person. The movement sensor detects the patient movement and also generates an alert message to the medical person. As comatose losses their sensation for urination, medical person needs to continuously monitor urine output, thus we are using ultrasonic sensor to check on urine level. Medical person can keep the track of patient using login to the system. The software IoT API we are using isThingSpeak.
\end{abstract}

Keywords-Comatose, IoMT, healthcare monitoring, ThingSpeak, Twilio

\section{INTRODUCTION}

In last decades health issues are raising day to day life at very high speed every day. One of the major health issue is a coma. Coma is a deep state of persistentsleep in which a person cannot be awakened; he fails to respond normally to painful stimuli, light, or sound; lacks a normal wake-sleep cycle; and does not initiate voluntary actions. Comatose arenot abletoknowinglyfeel,speakormove. Comas can be caused by various things such as- a severe injury to the head that hurts the brain, infections in the brain, brain damage due to lack of oxygen for too long, taking too much medicine (overdose) or other drugs, may be due to chemical imbalances in the body from other illnesses. Sometimes the person in a coma state can respond to the external environment by voluntary movements such as he may open his eyes in response to external impulse. Even though an individual in a coma state appears normal but they couldnotrespondtotheexternalcommands.Sincethephysical motionofpersistentvegetativestateindividualarerare,thereis a need for regular attention andcare. Technology will always makes things more simple and the human life much easier to survive. It affects the manyzones of life; one of the important region is medical field.Internet of Medical Things (IoMT) is an extension of the internet of things into the healthcare domain.Then IoMT is an ecosystem of interconnected sensors, wearable devices, medical devices, and clinical systems And applications that can connect to health care information technology systems using networking technologies.

Revised Manuscript Received on February 25, 2020

* Correspondence Author

Ankita Ramtirthkar*, Dept. of Electronicsand Telecommunication, Terna

college of engineering, Navi Mumbai ankitar08@gmail.com

Dr. Jyothi Digge, Dept. of Electronics and Telecommunication, Terna college of engineering, Navi Mumbai.

Prof.V.R.Koli Dept. of Electronics and Telecommunication, Terna college of engineering, Navi Mumbai virendrakoli@ternaengg.ac.in

(C) The Authors. Published by Blue Eyes Intelligence Engineering and Sciences Publication (BEIESP). This is an open access article under the CC BY-NC-ND license (http://creativecommons.org/licenses/by-nc-nd/4.0/)

It enables various healthcare applications to reduce healthcare costs, provide timely medical responses, and improves the quality of medical treatment. There are many applications of IoMT butone of the popular Example of IoMTis remote patient monitoring (RPM).

The comatose health history will be perceived and studied at any time at any place and by doctor with the help of wireless technology. With the help of technology we can permanently storepatient health information on the server. This paper delivers a health status assisting system that identifies human body parameters such as blood pressure, body temperature, body movement and more information on the IoT server through ThingSpeak. In emergency situations, this system automatically sent an alert SMS to the patient's caregivers, doctors if any strange data detected. Various sensors such as BP, temperature, acceleration and ultrasonic sensor, are used for a few seconds to collect body health parameter information for the proper treatment.

The use of Raspberry-Pi and IoT is adequate in health observation and this paper provides the awareness of both platforms. A popular Raspberry-Pi module offers a full Linux server on a small platform with IoT at a very low cost. Raspberry allows interface services and mechanisms via the general purpose $\mathrm{I} / \mathrm{O}$ interface. By using this combination, the proposed system is more effective, reliable, and flexible.

An IoT is a technique which connects the devices and offers human interaction to a better life. This paper, helps doctors for taking the right measurements of patient's health parameters and to give proper treatment to him at the appropriate time.

Here the proposed system shows a reliable uninterrupted health supervision system by the doctor, solution of patient's critical conditions anywhere in the world. The patient is carrying a set of body sensors to gather their different bodyparameters.

Presented paper is organized as follows. Section II discusses previous existingsystemsandtheirresearchwork done. In sectionIII proposed design methodology is explained. Section IV deals with the implementation of designed system. Section V presents simulation results of designed system. Finally, Section VI concludes the paper along with the further research andreferences.

\section{RELATEDWORK}

In this section, we surveyed existing systems and their research work dealing with analysis and monitoring of health parameters of coma patient.

There are numerousuninterrupted monitoring systems available in the medical field one of them is life scope VISMOPVM-2703.

\section{A. Lifescope Vismo Pvm-2703}

This deviceempowers to monitor ECG, pulse respiration, NIBP (non - invasive blood pressure amplifier), temperature. 
A large touchscreen enables quick and intuitive operation. But, it is very expensive. Also, the medical staff needs to manually record the parameters for every ten minutes which can cause human errors while recording[1].

Author [2], monitors different parameters of coma patient using PIC $\mu \mathrm{c}$ 8051. A zigbee[RF4CE] has been proposed and implemented to support the remote patient monitoring. The different sensors collect the medical data of patient and itgives feedback to the doctors. Zigbee module is connected to the $\mu \mathrm{c}$. zigbee is used for transfer values to the receiver side. Themain drawbackofthistechniqueis,itsendsdatatothenearestplace.

In [3], author proposes a health parameter monitoring system by using Raspberry-Pi. It mainly focuses on ECG only.it also uses zigbee, which sends data only to the nearest place.

In [4], author presents a health parameter analysis and monitoring of coma patients using wearable motion sensor technique. It uses LPC2148 ARM controller which needs extra hardware for connection to the internet, while this facility is inbuilt available in the raspberry-pi.

In [5], author describes model driven technology for automatic and cognitive IoT based system in the field of healthcare. Drawback of this technique is insufficient analysis and may be expensive due to prototype implementation.

\section{METHODOLOGY}

The proposed health monitoring system consists of different sensors which are divided into two categories. One is used for monitoring vitals of the comatose and second is used for detecting any physical changes occur in the comatose. Here, temperature and blood pressure are the two vitals recorded and monitored to understand health status of a comatose. The other two sensors are MEMS accelerometer sensor and Eye blink sensor which are used for detecting any physicalchangesthatoccurinacomatose.Thesesignalswhich provides information are recorded and monitored continuously to understand the body functioning. If the sets of these sensed signals which are outside normal ranges typically imply the need for some care or possible evacuation to a higher level of treatment during which we alert thedoctor.

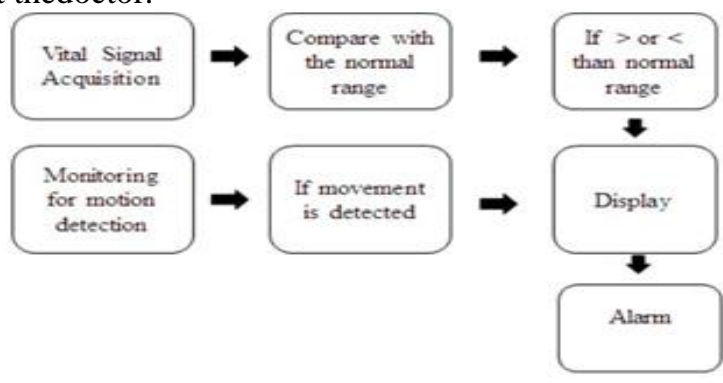

Fig.1- Methodology used in proposed system

\section{SYSTEMIMPLEMENTATION}

Thisdesignedsystemisconsistsoftwomodules, hardwarea nd software. The hardware module comprises transmitters and receivers which are nothing but different sensors and raspberry-pi module and the software modulecontains,Python, ThingSpeak, Twilio, and their interface. Developed monitoring system is evaluated for theperformance.

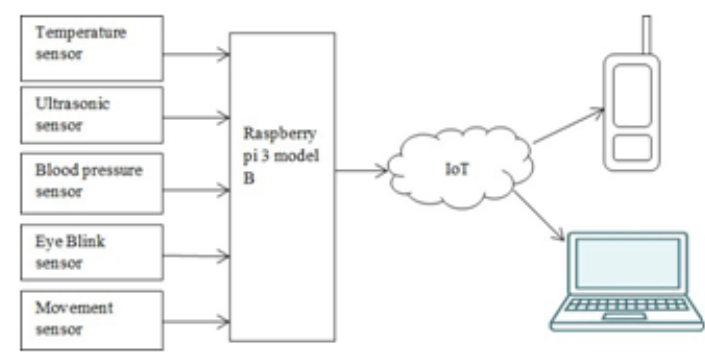

Fig. 2- Proposed system architecture

As shown in the Figure-2 architectural diagram of proposed model, one end of all sensors are connected to the patient's body and the other end is connected to the Raspberry-Pi board. Every sensors value is stored in the server and it will also display very latest values. The doctor/patient/guardian may see the patient's data along with their corresponding login details. A doctor will also see the patient's previous records and recommend changes in medicines and prescription, for further treatment.

\section{Proposed System}

A health observing system comprises of variety of sensors connected to the patient and they communicate that data via the processing unitto the server. In this project, Raspberry-Pi is acts as a data junction node as well as a processor. The patient and doctor smartphone or computers are used as a monitoring device.

As in figure-2, the sensors are used to measure the health parameters of patient after these parameters are acts as readings and finally converted into signals. These signals are provided for processing to Raspberry-Pi. Then Raspberry-Pi displays the information on a monitor and also stores the information over the cloud with the help of IoT. This information can be accessed by the doctor on his phone/computer and get the notification. Also there is facility provided to send an alert message to the doctor or patientcaregiver if any abnormal data is detected.

The workflow of the project is as- the sensors value are read and displayed on the monitor and stored in the cloud for future use.

Following is the flowchart of the described system-

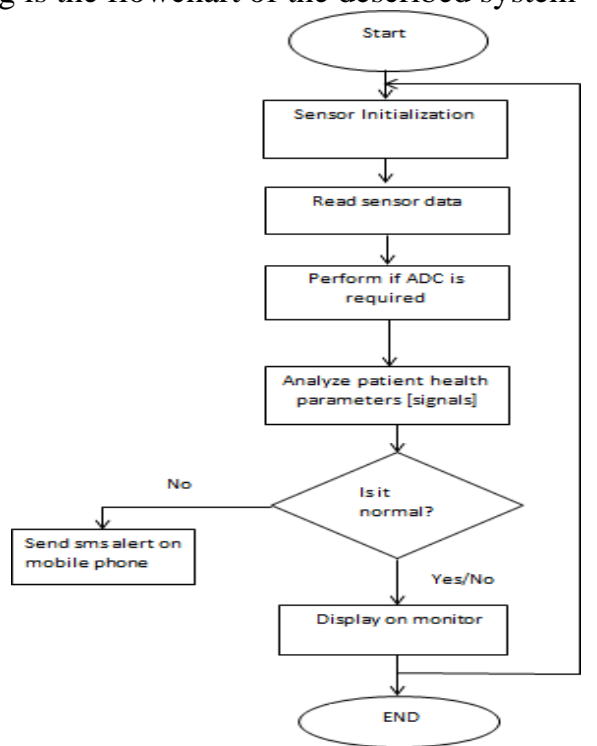

Fig no: 3 Flowchart of the designed system

Published By: 


\section{Hardware description-}

All sensors are interconnected with the Raspberry-Pi device and this device is connected to the IoT server, which provides services and controls over the network.

The hardware system include: -

- $\quad$ Blood pressure sensor: to know about the blood pressure rate of patient [SUNROM-1437]

- Temperature sensor: to measure the body temperature [LM 35]

- $\quad$ Eye blink sensor: to sense the movement of eye

- Ultrasonic sensor: to know about urine level [HCSR04]

- Accelerometer sensor: to detect change in body position [ADXL345].

The generated data of the sensors it transmitted to the Raspberry -pi through I2C and UART. Later this data is conveyed to the IoT server. Afterwards receiving the sensor's data, healthcare monitoring device will display these signals at regular intervals of time. Simultaneously it will process the data, accordinglyclinical test and the result should be in the safe range, otherwise the device is give out warning SMS to the patient caregiver or doctor or any specified physician.

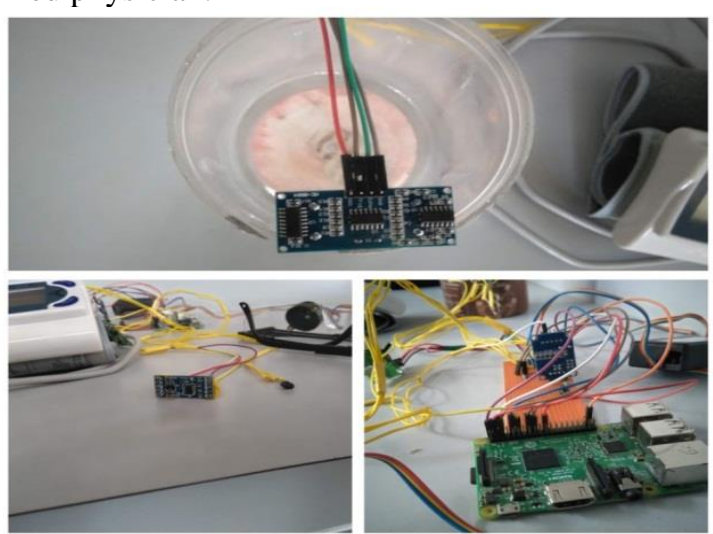

Fig4- Different sensors and their interconnections in the hardware

\section{Software description-}

ThingSpeak is an IoT based analytics platform package that allows you to combine, envision and evaluate live data streams in the cloud. ThingSpeak deals with the instantaneous visualization of data sent by your devices to ThingSpeak. Using ThingSpeak you can perform online analysis and processing of the data as it comes in.ThinkSpeak itself acts as a 'cloud' interface.ThingSpeak has many features such as- collection of data in private channels and sharing that data on to the public channel, MATLAB analysis and visualization, App integration and worldwide community.

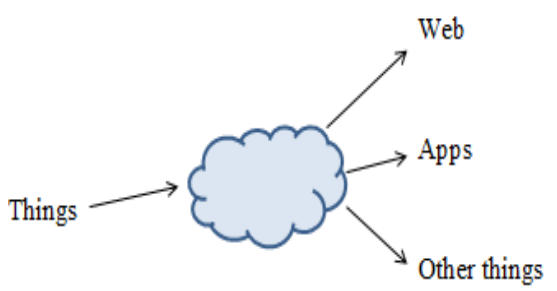

Fig.5 -Representation of ThingSpeak API
Twilio is cloud communication based platform. Twilio API enables to make and receive voice and video calls, send and receive text as well as voice messages, and perform other communication functions for global scale.

\section{Features of proposed system-}

1) $24 * 7$ monitoring of comapatient.

2) Continuous recording of patient data after particular time period.

3) Cost islow.

4) Power consumptionless.

5) No need of human attention for continuous observation of comapatient.

6] High speed communication between doctor and patient through Thingspeak cloud as well as Twilio.

7] The doctor can easily control on the patient through world wide, anywhere any time.

8] System is completely portable. 9] It will help to reduce the time.

10] We can store all data's in the database, so that we will not lose them.

\section{Weakness -}

1) Movement sensor should be moresensitive.

2) Blood pressure sensor requires approx. 60+ sec to detect anddisplay.

3) Require to reset system when treatment isdone.

4) On input side internet facility is compulsoryrequired.

\section{RESULT AND DISCUSSION}

The graphical representation of information of the patient which means exactly health status is established i.e. body temperature, blood pressure are as showed up in the fig-6 which is observed by signing into the Thingspeak server through a personal computer which has Date in $\mathrm{x}$ axis and the parameter in y-axis. If there is any changes occur in body position, eye movement and urine level of comatose then an alert SMS is send to the doctor or any medical person with the help of Twilio. Fig-7 shows the different alert sms received by doctor. So it is very useful for patient's to give first aid at any time and to report a clear notification of patient database health status in graphicalform.

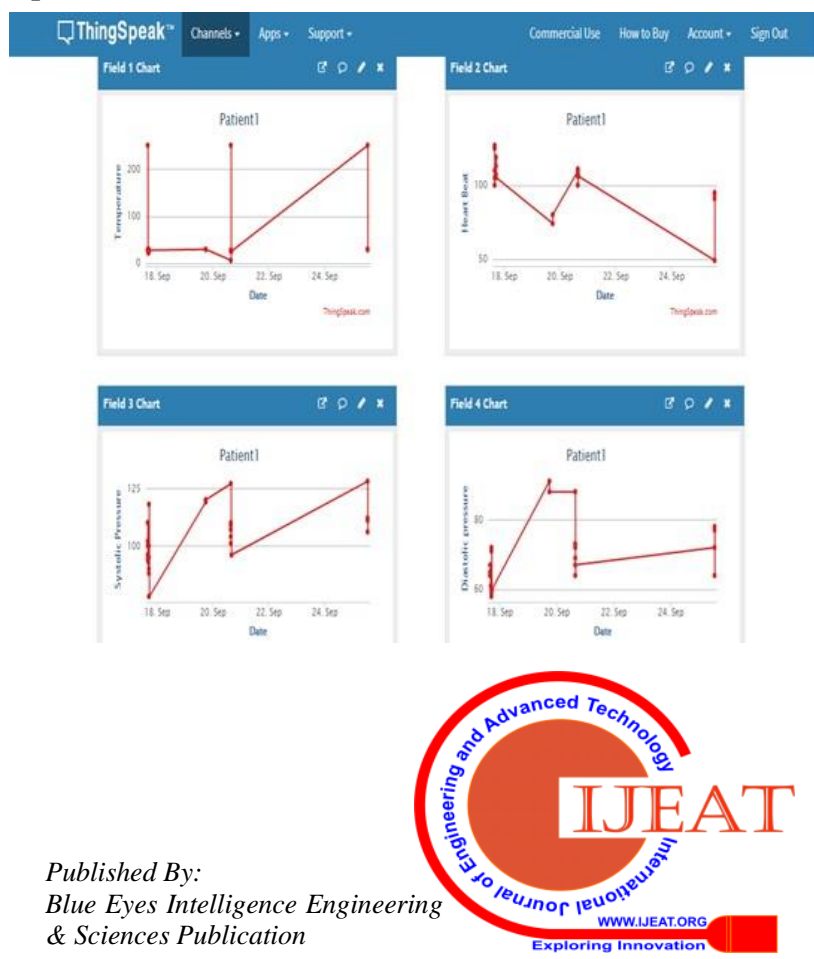




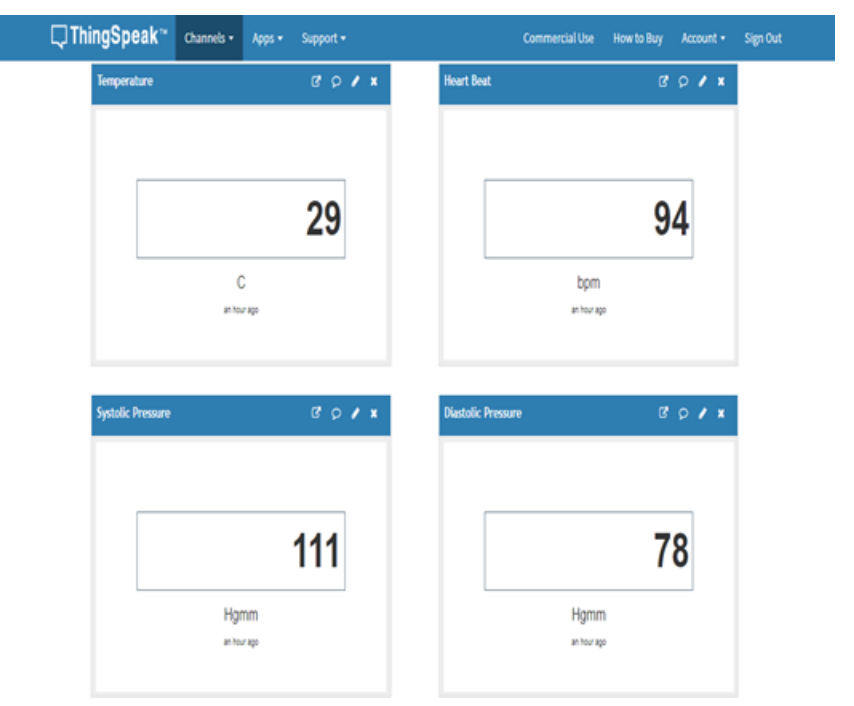

Fig-6: The biomedical status of the patient in the Thingspeak channel

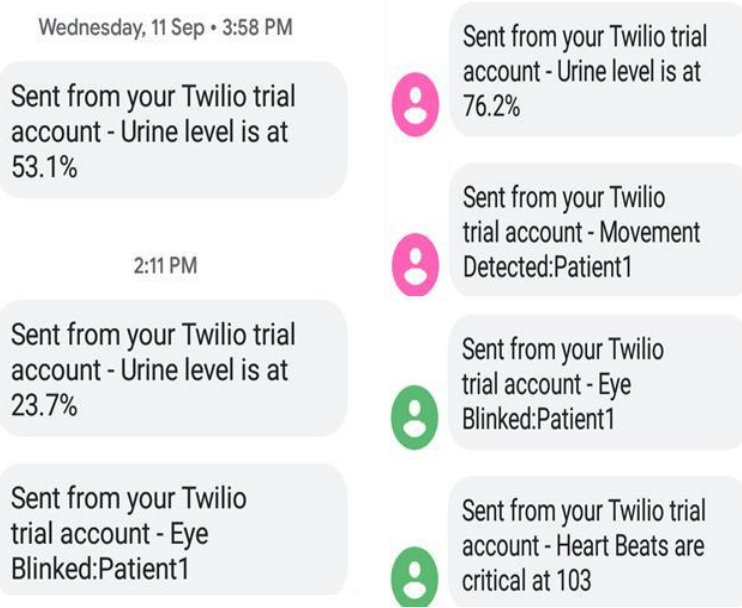

Fig-7: Screenshots of Alert SMS

\section{CONCLUSION}

The aim of our proposed system is to build easily accessible design that the patient's critical information is conveyed quicklyto the doctor is achieved.The designed modelleads to the better and effective health care service to comatose and the collected data is networked worldwide with the help of internet and communication which provide a quick response. The IoMT market involves variety of smart devices, such as wearable and medical/vital monitors, in the home, or hospital; and associated real-time location, telehealth and other services. So with the help of these devices doctor can easily examine his patient at anytime, anyplace.

In this proposed system vital parameters such as B. P., temperature are monitored. Movement sensor and eye blink sensor is used to detect motion of body parts of comatose. Ultrasonic sensor is used for urine level monitoring. Also the designed system is affordable to the patients.

\section{FUTURE SCOPE}

1. For further implementation, in emergency situations, this system can automatically send a warning message or call to alert the nearest hospital as well as to the ambulanceif any abnormal data is identified inmonitoring.

2. The advanced development for the designed model is to add more parameters for monitoring the health status of patient.

3. In future improvement to this designed model is to include MEDIBOX. This system can be used by theparalyzed patients by sending or giving reminder alert to take their medicines or dosage on time.

4. Another extension to this system is to add web camera, after that anyone can monitor patient worldwide at any time.

\section{REFERENCES}

1. Dr.R.JosphineLeelaM.E , P K.Hamsageetha , P.Monisha, S.Yuvarani, "Body Movement and Heart Beat Monitoring For Coma Patient Using IoT", International Journal of Innovative Research in Science, Engineering and Technology, Vol. 7, Special Issue 2, March2018.

2. Geethanjali R., MajidhaFathima K. M., Harini S., Sabitha M., "Health monitoring for coma patients", International Journal of Emerging Research \& Development, Volume 2, Issue 3,2019.

3. S. Sandeep, Dr. P. Esther Rani, G.Sumalatha, "Monitoring of Health Parameters by Using Raspberry Pi”, International Journals of Advanced Research in Computer Science and Software Engineering, (Volume-8, Issue-4),2018.

4. SnehaChowdaryKoganti, Dr. H N Suma, Appaji M. Abhishek "Analysis and Monitoring of Coma Patients using Wearable Motion Sensor System", International Journal of Science and Research (IJSR), Volume 4 Issue 9, September2015.

5. EmnaMezghani, Ernesto Exposito, and Khalil Drira.,"A ModelDriven Methodology for the Design of Autonomic and Cognitive IoT-Based Systems: Application to Healthcare", IEEE transactions on emerging topics in computational Intelligence, VOL. 1, NO. 3, JUNE2017.

6. http://www.Twilio.com

7. http://thingspeak.com 